\title{
Beitrag zur Wissenschafts- und Institutionengeschichte der Wiener Germanistik
}

Elisabeth Grabenweger: Germanistik in Wien. Das Seminar für Deutsche Philologie und seine Privatdozentinnen (1897-1933). Göttingen: de Gruyter 2016 (Quellen und Forschungen zur Literatur- und Kulturgeschichte, Bd. 85/319), $280 \mathrm{~S}$.

Wie war es möglich, dass in Wien in den 1920er Jahren drei Privatdozentinnen an der Germanistik lehrten, während an allen anderen Instituten des deutschen Sprachraums zeitgleich keine Frau oder eben nur eine Frau zur Habilitation zugelassen wurde (S. 3)? Diese Frage stellt die Wiener Germanistin Elisabeth Grabenweger in ihrer Monographie, einer "geringfügig veränderte[n] Fassung (S. 5) ihrer 2014 von der PhilologischKulturwissenschaftlichen Fakultät der Universität Wien angenommenen Dissertationsschrift. Um die Frage beantworten zu können, rekonstruiert Grabenweger mithilfe von Archivmaterialien, zeitgenössischen Publikationen, Zeitungsberichten, Gesetzestexten und Forschungsliteratur die Wissenschafts- und Institutionengeschichte der Wiener Germanistik nicht nur von 1897 (bzw. von der Zulassung der Frauen an der philosophischen Fakultät) bis 1933 (als sie ihre akademische Lehrtätigkeit vorläufig unterbrachen), sondern beginnt ihre Untersuchungen schon mit dem Jahr 1848. Die Ausnahmestellung des Wiener Germanistikinstituts wird dabei sowohl im Hinblick auf andere Germanistikinstitute im deutschsprachigen Raum als auch mit Rücksicht auf alle anderen Studienrichtungen der damaligen philosophischen Fakultät hervorgehoben.

Nach einer kurzen »Einleitung « (S. 1-6) folgt das erste und zugleich längste Kapitel »Die Verfasstheit der Wiener Germanistik «. Auf etwa achtzig Seiten (S. 7-88), in vier Unterkapiteln, ${ }^{1}$ rekonstruiert die Autorin sowohl die 
fachliche als auch die institutionelle Entwicklung des Gemanistikinstituts, einschließlich dessen Personalpolitik. Die vorgeschlagene Periodisierung geht von drei Entwicklungsetappen aus. Das Wiener Germanistikinstitut wurde 1848 gegründet; bis 1868 dauerte die »Konsolidierungsphase des Faches « (S. 7), in der die Lehrstühle direkt vom Ministerium für Kultus und Unterricht ohne Fakultätsvorschlag besetzt wurden. Wegen des Mangels an akademisch ausgebildeten Germanisten prägten die erste Etappe die nicht-habilitierten Privatgelehrten oder Wissenschaftler anderer Fachrichtungen, wie der germanistische Autodidakt und Bibliothekar an der Wiener Hofbibliothek, der griechisch-orthodoxe Theodor Georg von Karajan, der protestantische klassische Philologe Karl August Hahn aus Deutschland und der Privatgelehrte Franz Pfeiffer, ein ehemaliger Medizinstudent aus der Schweiz. Die »nationale und konfessionelle Offenheit« (S. 8) endete zwanzig Jahre nach der Gründung des Germanistikinstituts. Eine wichtige Zäsur und den Beginn der zweiten Etappe bildeten zwei Ereignisse im Jahr 1868: die Errichtung der Lehrkanzel für die neuere Literatur und der erste erfolgreiche Versuch der Fakultät, sich in das Nachbesetzungsverfahren zu involvieren. Es wurde sogar ein Mechanismus eingeführt, »nach dem der Favorit des scheidenden Lehrstuhlinhabers dessen Nachfolge antrat«(S. 10).

Die Entwicklung der Wiener Germanistik kann freilich nicht unabhängig vom historischen Kontext betrachtet werden. Die Niederlage im Deutschen Krieg - der siebenwöchigen militärischen Auseinandersetzung zwischen Preußen und preußischen Verbündeten einerseits und dem Deutschen Bund unter Führung Österreichs andererseits (1866) - sowie der Österreichisch-Ungarische Ausgleich (1867) zwangen Österreich dazu, seine Kulturpolitik zu modifizieren. Vielleicht liegt auch darin der Grund, dass die Berufung neuer Professoren nach 1868 auch den nationalen Anforderungen des Ministeriums entsprechen musste. Das vorherrschende Muster fasst Grabenweger folgenderweise zusammen: »Österreicher - Schüler des Vorgängers - Katholik«(S. 76). Die »strikte Nachbesetzungspraxis« kennzeichnete die Wiener Germanistik bis ins Jahr 1912, als die "problemfreie Kontinuität« (S. 16) und die »konfliktfreie Traditionsbewahrung» (S. 231) ein Ende fanden.

Die neuen wissenschaftlichen Tendenzen in der Germanistik, d.h. "philosophische, kunsttheoretische und formalästhetische Ansätze«(S. 47), die sich auf Kosten des traditionellen philologischen Zugangs durchzusetzen 
begannen, sowie der allmähliche Verlust des elitären Status, den die Universitäten früher gehabt hatten, bereiteten der 1868 postulierten Entwicklung ein Ende. Die Berufung von Walther Brecht aus Augsburg hält Grabenweger für eine Wende, die die Habilitation der drei Privatdozentinnen ermöglichte. Die Arbeit und das Wirken des für das ältere Fach habilitierten Ordinarius für die neuere Literatur wird im Unterkapitel »Philologie und moderate Geistesgeschichte - Walther Brecht am neugermanistischen Lehrstuhl in Wien 1914-1926 (S. 40-75) dargestellt. Paradoxerweise wurde er, der »als öffentlich Unbekannter nach Wien kam und bis heute als einer der `wenigst berühmt gewordenen Germanisten ` der ersten Hälfte des 20. Jahrhunderts« gilt, zu »einem ausgleichenden Vermittler österreichischer Literatur, zu einem Wissenschaftler, der die divergierenden methodischen Richtungen der 1910er und 1920er Jahre anzunähern trachtete, und zum ausgewiesenen Förderer einer ganzen Generation von Neugermanisten « (S. 40).

Im letzten Unterkapitel wird die Antwort auf die eingangs gestellte Frage gegeben. Die Habilitation der drei Frauen hatte wenig oder nicht nur mit ihrer Emanzipation in der akademischen Gesellschaft zu tun. Ganz im Gegenteil. Da die Zahl der Studierenden kontinuierlich anstieg und vor allem wegen der staatlichen Sparmaßnahmen die Zahl der Professuren nahezu gleich blieb, übernahmen die Privatdozenten einerseits immer mehr Unterricht. Andererseits hatten sie immer weniger Chancen, Extraordinarien oder Ordinarien zu werden. »Die finanzielle Schlechterstellung und die schwindende Wahrscheinlichkeit einer akademischen Karriere führten zu einem massiven Prestigeverlust der Privatdozentur «, so Grabenweger (S. 86). Der Prestigeverlust gehöre zur Erklärung des Umstands, dass auch Frauen zur Privatdozentur zugelassen wurden. Die Habilitationen wurden von Brechts Nachfolger Paul Kluckhohn weiter gefördert, aber bei Josef Nadler, der nach Kluckhohns Rücktritt die Neugermanistik leitete, habilitierte während seiner 14-jährigen Amtszeit »nicht nur keine einzige Frau«, sondern überhaupt kein einziger Wissenschaftler (S. 88).

Während Grabenweger im ersten Kapitel die Entwicklung der Wiener Germanistik hauptsächlich anhand von aus den Archivquellen, zeitgenössischer Publizistik und der Korrespondenz entnommenen Informationen rekonstruiert, bedient sich die Autorin in den nächsten Kapiteln, die die Einzeldarstellungen der ersten in Wien habilitierten Germanistinnen umfassen, auch biographischer und hermeneutischer Methoden. Im zweiten Kapitel, »Frauen als Autorinnen und Wissenschaftlerinnen und die Neuere deutsche Literaturwissenschaft - Christine Touaillon (1878-1928)«, beschäftigt sich die Autorin mit der ersten österreichischen Germanistin, die 1921 als Privatdozentin für neuere deutsche Literaturgeschichte zugelassen 
wurde (S. 89-138). Das Kapitel beginnt mit einer kurzen Darstellung ihrer Bildungslaufbahn, Veröffentlichungen und ihrer öffentlichen Tätigkeit (S. 89-92). Das erste von vier Unterkapiteln ${ }^{2}$ zeigt nicht nur Touaillons Bemühungen, die Venia Legendi zu erlangen, sondern gibt auch einen Überblick über die an den Universitäten in Graz und Wien geführten Diskussionen, ob Frauen und Männer unter den gleichen Bedingungen zur Habilitation zugelassen werden sollten. Mit ihren Forschungen weckte Touaillon Interesse für die Rolle der Frauen in der Literatur, gab »den Anstoß zur universitären Auseinandersetzung mit Literatur von Frauen « (S. 134f.) und ermunterte viele, Dissertationen über Schriftstellerinnen zu verfassen. Touaillons Erfolg erklärt Grabenweger mit ihrer »wissenschaftliche[n] Spezialisierung", bzw. mit der Tatsache, »dass sie sich nicht in den Kanon der ১bedeutenden< Forschungsthemen einschrieb und damit nicht mit ihren männlichen Kollegen in Konkurrenz trat «(S. 234). Die Marginalisierung von Touaillons Forschungstätigkeit führt sie neben der Themenwahl auch auf ihre politische Nähe zur Sozialdemokratie in einer zunehmend konservativen Umgebung der Wiener Universität zurück (S. 136).

Das dritte Kapitel, »Literaturwissenschaft und Geistesgeschichte - Marianne Thalmann (1888-1975)« (S. 139-182), ist der »ambivalenteste[n] Figur unter den Wiener Privatdozentinnen « (S. 234) gewidmet. ${ }^{3}$ Nach einer Darstellung der Bildungslaufbahn und Berufstätigkeit folgt ein Überblick über die von Thalmann behandelten Themen. Die ambivalente zeitgenössische Rezeption Thalmanns - die sich "von hoher fachlicher Wertschätzung bis hin zu wissenschaftlicher Diskreditierung (S. 143) spannte - wird exemplarisch an zwei Werken im ersten und zweiten Unterkapitel dargestellt. Obwohl Thalmann den Begriff »Trivialroman « als Fachterminus in die Wissenschaft eingeführt hatte, unterschied sich ihr Forschungsinteresse von dem anderer Trivialliteraturforscher dadurch, dass sie den Trivialroman »einzig und allein als >Vorläufer des romantischen Romanes«" (S. 152) verstand. Grabenweger sieht das als eine erfolgreiche Strategie, »die darauf abzielte, als Frau mit einem Randforschungsgebiet eher habilitiert zu werden als mit einem Thema, das im Zentrum des germanistischen

2 Zwischen Universität und Staatsverfassung - Habilitationsverfahren in Graz und Wien (S. 93-102); Literatur-, Kultur- und Sozialgeschichte - Der deutsche Frauenroman des 18. Jahrhunderts (1919) (S. 102-121); Kanon und Geschlecht (S. 121-129); Themenwahl und akademische Karriere (S. 129-138).

3 Darstellung statt Erkenntnis? - Der Trivialroman und der romantische Roman. Ein Beitrag zur Entwicklungsgeschichte der Geheimbundmystik (1923) (S. 144-164); Konservativ-pessimistische Zeitdiagnose einer Intellektuellen - Die Anarchie im Bürgertum. Ein Beitrag zur Entwicklungsgeschichte des liberalen Dramas (1932) (S. 164-175); Wiener Karriere und Weggang in die USA (S. 175-182). 
Interesses stand und somit von Männern besetzt war « (S. 153). Erst nach der Habilitation verfasste sie Schriften über Johann Wolfgang Goethe und Adalbert Stifter, also über Themen, die »den innersten Kanon der germanistischen Forschungsgegenstände" gehörten (S. 235). Mit ihrem Werk Die Anarchie im Bürgertum näherte sich Thalmann »den antidemokratischen, ansonsten fast ausschließlich von Männern getragenen intellektuellen Ideen der >Konservativen Revolution « (S. 235). Das Kapitel endet mit Thalmanns letzten Jahren an der Wiener Universität und ihrer Karriere in den USA.

Im letzten Kapitel, »Deutsche Philologie als Germanen- und Volkskunde - Lily Weiser (1898-1897) « (S. 183-230), wird das akademische Schicksal der dritten an der Wiener Germanistik habilitierten Frau dargestellt, die als erste die Venia Legendi für Germanische Altertums- und Volkskunde erhielt. Im Unterschied zu Christine Touaillon und Marianne Thalmann, deren Habilitationsthemen mehr oder weniger "an der Peripherie des wissenschaftlichen Interesses angesiedelt war[en] «(S. 186), war Weisers Thema über die altgermanischen Jünglingsweihen und Männerbunde »die zeitgenössisch meist diskutierte Frage der Germanenkunde« (S. 186). Im ersten Unterkapitel erörtert Grabenweger die Rolle Rudolf Muchs, jenes Germanisten, »in dessen Gravitationsbereich Weiser studierte« und der die Germanistik als die »Wissenschaft von den Germanen und vom deutschen Volk« (S. 187) verstand. Im zweiten Unterkapitel analysiert sie vor allem anhand von Weisers Dissertation ihr »Verständnis von germanistischer Volkskunde« (S. 186) und belegt, dass Weiser der Weltauffassung ihres Betreuers folgte. Die Analyse ihrer Habilitationsschrift und des Motivs der Männerbünde in der Germanenkunde folgt im dritten Unterkapitel. Im letzten Unterkapitel werden »Ideologien und Netzwerke, Konkurrenz- und Machtverhältnisse im Bereich der Volkskunde an der Wiener Universität betrachtet, um die Position Weisers in dem zuvor beschriebenen Subfeld der Germanistik zu bestimmen« (S. 186). Weisers Karriere an der Universität Wien endete schon nach einem Semester, weil sie mit ihrem Mann nach Oslo zog. Da Much neben Weiser noch einige Habilitationen erfolgreich betreut hatte, gelang es ihm völlig »die Volkskunde in seine, nämlich die german(ist)ische Richtung zu ziehen « (S. 216) und eine »Schule« zu schaffen, die sich gegenüber der Konkurrenz durchsetzte und die »zu einer universitätspolitisch mächtigen Ideologie- und Forschungsgemeinschaft»

4 Altertums- und Germanenkunde - Rudolf Much (1862-1936) (S. 187-197); Volkskunde existiert nur als Germanenkunde - Jul. Weihnachtsgeschenke und Weihnachtsbaum (1923) (S. 197-206); Archaische Potenzfeiern als Ursprung der deutschen Kultur - Altgermanische Jünglingsweihen und Männerbünde (1927) (S. 206-216); Konkurrenzen und Netzwerke (S. 216-230). 
(S. 236) wurde, schlussfolgert Grabenweger. Das Buch endet mit einem sechsseitigen Resümee (S. 231-236) und einem Anhang (S. 237-280), der ein Literatur- und Quellenverzeichnis sowie ein Personenregister enthält.

In den letzten Jahren ist ein verstärktes wissenschaftliches Interesse für die Geschichte von Universitäten und wissenschaftlichen Instituten zu verzeichnen. Im Mittelpunkt stehen dabei institutionelle Rahmenbedingungen, Curricula, Lehrkräfte und Studenten. Elisabeth Grabenweger folgt dem Trend, doch ihre Arbeit weist einige Besonderheiten auf, vor allem was den Zugang zum Thema betrifft. Bei der Rekonstruktion der Entwicklungsgeschichte der Wiener Germanistik wird nämlich ein wichtiger Aspekt einbezogen - die Geschlechtergeschichte. Damit ist ihre Monographie ein bedeutender Beitrag zu einem Forschungsfeld, das sich in neuer Perspektive auf das Verhältnis von Wissen und Gender konzentriert: »auf jene relativ raren Fälle, in denen Frauen Zugang zu Institutionen erkämpften oder [... ] sich alternative Räume für gelehrte Kommunikation schufen «. ${ }^{5}$

Die geschickte Verknüpfung der fachlichen Porträts der drei Privatdozentinnen mit der Wissenschafts- und Institutionengeschichte macht die vorliegende Monographie attraktiv und nützlich sowohl für diejenigen, die Interesse an der Geschichte der Germanistik und der Geschlechtergeschichte haben, als auch für alle, die sich mit der Geschichte der Universitäten beschäftigen. Doch geht es nicht nur um einen Beitrag zur Wissenschaftsgeschichte: Zahlreiche Details öffnen Leserinnen und Lesern facettenreiche Einblicke in den `Zeitgeist der Epoche; das Buch ist somit auch ein bedeutender Beitrag zur Kulturgeschichte Österreichs. Zieht man all das in Betracht, lässt sich abschließend sagen, dass Elisabeth Grabenweger echte Pionierarbeit geleistet hat. 